\title{
Suppressive effects of quercetin on hydrogen peroxide-induced caveolin-1 phosphorylation in endothelial cells
}

\author{
Akari Kondo-Kawai, ${ }^{1,2,3}$ Tohru Sakai, ${ }^{2}$ Junji Terao, ${ }^{1,4}$ and Rie Mukai ${ }^{1,5, *}$ \\ ${ }^{1}$ Department of Food Science, Graduate School of Biomedical Sciences and ${ }^{5}$ Department of Food Science, Graduate School of Technology, \\ Industrial and Social Sciences, Tokushima University, Tokushima, Tokushima 770-8513, Japan \\ 2Department of Public Health and Applied Nutrition, Institute of Biomedical Sciences, Tokushima University Graduate School, Tokushima, \\ Tokushima 770-8503, Japan \\ ${ }^{3}$ Laboratory of Nutritional Science, Shikoku Junior College, Tokushima, Tokushima 771-1192, Japan \\ ${ }^{4}$ Faculty of Clinical Nutrition and Dietetics, Konan Women's University, Kobe, Hyogo 658-0001, Japan
}

(Received 27 November, 2020; Accepted 19 December, 2020; Published online 3 April, 2021)

Caveolin-1 is a major protein of the caveolae structure in vascular endothelial cell membrane. Phosphorylation of caveolin-1 is one of the initial events leading to exacerbation of vascular permeability caused by oxidative stress. Although quercetin is known to be an anti-atherosclerosis factor that acts as a dietary antioxidant, little is known about its role in the regulation of caveolin-1 phosphorylation. In this study, we investigated the inhibitory effect of quercetin on hydrogen peroxide-induced caveolin-1 phosphorylation in human umbilical vein endothelial cells. Quercetin inhibited caveolin-1 phosphorylation in cells pretreated with quercetin for $24 \mathrm{~h}$ and then exposed to hydrogen peroxide. However, quercetin 3-O- $\beta$-glucuronide, a conjugated metabolite of quercetin, did not exert this inhibitory effect. Exposure to hydrogen peroxide increased vascular permeability and reduced mRNA expression of the intercellular adhesion protein, vascular endothelial cadherin (VE-cadherin). By contrast, pretreatment with quercetin suppressed the increase in vascular permeability and decreased VE-cadherin expression. These results indicate that deconjugated quercetin can play a role in the prevention of altered vascular permeability under oxidative stress by suppressing caveolin-1 phosphorylation. Thus, dietary quercetin may be beneficial for the maintenance of endothelial cell function.

Key Words: quercetin, caveolae, caveolin-1, hydrogen peroxide, vascular endothelium

$\mathrm{C}$ ardiovascular disease has provoked risk for premature death worldwide. $^{(1,2)}$ It was estimated 422.7 million people was suffered from cardiovascular disease in 2015 , and was estimated 17.9 million deaths worldwide in 2015. It was comprising $31 \%$ of all global deaths. ${ }^{(1,3)}$ Atherosclerosis is one of the factors that cause coronary artery disease and cerebrovascular disease. Atherosclerosis results in dysfunction of the vascular endothelium, formation of fatty streak in blood vessels, and plaque fibrosis. ${ }^{(4)}$ Basic diet control and exercise therapy are needed for preventing atherosclerosis. ${ }^{(5)}$ In addition to this, it has been suggested that several bioactive within dietary food can be is one of the solution for preventing atherosclerosis. ${ }^{(6,7)}$

Endothelial cells play a central role in maintaining homeostasis of vascular function..$^{(8,9)}$ Tight junctions and adherens junctions suppress the permeation of substances through the gap of neighboring vascular endothelial cells. However, the impairment of cell-cell adhesion in inflamed tissues enhances vascular permeability, ${ }^{(10,11)}$ and the resulting invasion of lipoproteins and/or plasma components into the vessel walls accelerates vascular endothelial dysfunction. Vascular endothelial cells are densely populated with caveolae, which are flask-shaped invaginations measuring $50-100 \mathrm{~nm}$ in diameter. $^{(12)}$ Caveolae are rich in sphingolipids and cholesterol, and are implied to serve as a platform for endocytosis and signal transduction, ${ }^{(13-16)}$ particularly in the cell membrane. Caveolin-1 (Cav-1), a major protein component of caveolae, is involved in the regulation of lipoprotein transcytosis, vascular inflammation, ${ }^{(17)}$ and progression of atherosclerosis. ${ }^{(18)}$

Phosphorylation of Cav-1 has been reported to increase the endocytosis of plasma albumin and contribute to vascular permeability by enhancing vascular endothelial cadherin (VE-cadherin) endocytosis. ${ }^{(19-21)}$ Furthermore, phosphorylation of Cav-1 in the endothelium was shown to be enhanced in response to oxidized low-density proteins (ox-LDL) and hydrogen peroxide $\left(\mathrm{H}_{2} \mathrm{O}_{2}\right) \cdot{ }^{(22,23)}$ It is therefore likely that the inhibition of Cav-1 phosphorylation is involved in the protective action of antioxidative food factors in the endothelium.

Flavonoids, which are composed of diphenyl propane structure, are distributed ubiquitously in the plant kingdom and have been suggested to exert a wide variety of health promoting effects. (24-26) It has already been reported that higher flavonoid intake lowers mortality due to cardiovascular diseases. ${ }^{(27)}$ Moreover, it is known that the amount of flavonoid intake is inversely correlated with the incidence rate of cardiovascular diseases. ${ }^{(28)}$ Quercetin (3,3,4,5,7-pentahydroxyflavone; Q), a flavonol-type flavonoid commonly present in fruits and vegetables, is a potential candidate for use in preventing the onset of cardiovascular diseases owing to its anti-oxidative or anti-atherosclerosis functions. ${ }^{(29-31)}$

Although Q mainly exists as a glucoside in plants, ${ }^{(32)}$ it is hydrolyzed in epithelial cells of the small intestine during absorption, ${ }^{(33)}$ and the resulting aglycone is further converted into various metabolites, including glucuronides, sulfates, and/or their $O$-methyl derivatives (isorhamnetin and tamarixetin) in the small intestine and liver. ${ }^{(33)}$ Kawai et al. ${ }^{(34)}$ and Ishisaka et al. ${ }^{(35)}$ reported that Q 3-O-glucuronide (Q3GA), a major conjugated Q metabolite, is converted to its aglycone form by the action of macrophages. It is likely that Q glucuronide metabolites present

Department of Food Science, Graduate School of Biomedical Sciences, Tokushima University is the previous affiliation for Akari Kondo-Kawai, Junji Terao, and Rie Mukai. They continued this work at the present affiliation.

*To whom correspondence should be addressed.

E-mail: rmukai@tokushima-u.ac.jp 
in the plasma act as anti-atherosclerotic agents in the aorta after it is converted to its aglycone form. ${ }^{(36-38)}$ In a previous study, ${ }^{(39)}$ we investigated the effects of Q glucuronide metabolites and its aglycone on ox-LDL-induced expression of Cav-1 in human umbilical vein endothelial cells (HUVECs). The purpose of this study was to evaluate the protective role of $\mathrm{Q}$ against endothelial dysfunction, with a focus on suppression of Cav-1 phosphorylation using a $\mathrm{H}_{2} \mathrm{O}_{2}$-exposed HUVECs system.

\section{Materials and Methods}

Reagents. Q dehydrate, $\mathrm{H}_{2} \mathrm{O}_{2}$, and anti-Cav-1 (c3237) were purchased from Sigma Aldrich Co. (St. Louis, MO). Antiglyceraldehyde-3-phosphate dehydrogenase (GAPDH, 14C10) and anti-phospho-Fyn (D49G4) were obtained from Cell Signaling Technology (Beverly, MA). Anti-phospho-Cav-1 (sc-14037) and anti-Fyn (sc-16) antibodies were obtained from Santa Cruz Biotechnology (Santa Cruz, CA). Q3GA and myricetin (M) (1127S) were obtained from Extrasynthese (Genay, France). Flavone (F) was obtained from Nacalai Tesque (16012-31) (Kyoto, Japan). Luteolin (L) was obtained from LKT Laboratories (L8377; Saint Paul, MN) and 8-prenyl Q was synthesized as previously described by Kawamura et al. ${ }^{(40)}$

Cells and culture. HUVECs were purchased from Lonza Japan (Tokyo, Japan). HUVECs were cultured in endothelial cell growth medium EGM-2 (cc-3156) mixed with SingleQuotsTM (cc-4176) (Lonza, Basel, Switzerland) and maintained at $37^{\circ} \mathrm{C}$ in a humidified atmosphere containing $5 \% \mathrm{CO}_{2}$. Cells were used at passages from \#3 to \#10.

Real-time reverse-transcription polymerase chain reaction (RT-PCR). HUVECs $\left(6 \times 10^{4}\right.$ cells/well $)$ were plated on 24well plates and incubated for $24 \mathrm{~h}$. Cells were treated with $\mathrm{H}_{2} \mathrm{O}_{2}$ and Q-related compounds. To estimate the effects of flavonoids, they were added to the cells before $\mathrm{H}_{2} \mathrm{O}_{2}$ treatment. Briefly, cells treated with flavonoids were washed with Hank's balanced salt solution (HBSS) and transferred to fresh medium containing $\mathrm{H}_{2} \mathrm{O}_{2}$. Total RNA was isolated from HUVECs using ISOGEN (Nippon Gene, Toyama, Japan). mRNA expression was determined by real-time reverse-transcription polymerase chain reaction (real-time PCR), as previously described. ${ }^{(41)}$ The primers used in this study are indicated in Table 1.

The relative gene expression levels in each sample compared to the control were calculated using the comparative $\mathrm{Ct}$ method $(\Delta \Delta \mathrm{CT})$. The gene expression levels of target genes in each sample were normalized to that of GAPDH $\Delta \Delta \mathrm{CT}$ value.

Western blotting. HUVECs were seeded on $60 \mathrm{~mm}$ dishes $\left(5 \times 10^{5}\right.$ cells/dish $)$ and incubated for $24 \mathrm{~h}$. These cells were treated with Q-related compounds. The treated cells were washed with HBSS and fresh medium containing $\mathrm{H}_{2} \mathrm{O}_{2}$ added. After incubation for $24 \mathrm{~h}$, the cells were washed twice with HBSS and lysed with lysis buffer $[50 \mathrm{mM}$ Tris- $\mathrm{HCl}$ ( $\mathrm{pH}$ 8.0), $200 \mathrm{mM} \mathrm{NaCl}, 20 \mathrm{mM}$ EDTA, 1\% sodium dodecyl sulfate polyacrylamide (SDS), $0.5 \%$ sodium deoxycholate, $0.01 \%$ Nonidet P-40, phosphatase inhibitor tablet (Phos STOP), and protease inhibitor tablet (complete EDTA-free)]. Protein concentrations were determined using the bicinchoninic acid
(BCA) protein assay (Pierce; Thermo Scientific, Waltham, MA). The protein samples were boiled with reducing buffer (Nacalai Tesque) for 5 min. The samples were separated by 15\% SDS gel electrophoresis (SDS-PAGE). Proteins were transferred onto Immobilon P PVDF transfer membranes (Millipore, Bedford, MA), followed by blocking of non-specific binding with a commercial blocking buffer (Blocking One for GAPDH, Cav-1 and Fyn or Blocking One $\mathrm{P}$ for phosphor Cav-1 and phosphor Fyn; Nacalai Tesque) for $1 \mathrm{~h}$. After washing with Tris-buffered saline containing $0.05 \%$ Tween 20 (TBST), the membranes were incubated with anti-Cav-1 antibody, anti phospho-Cav-1 antibody, anti-Fyn antibody, anti-phospho-Fyn antibody, or antiGAPDH antibody for $1 \mathrm{~h}$ at room temperature. After washing three times with TBST, the membranes were incubated for $1 \mathrm{~h}$ at room temperature with a secondary antibody. After washing with TBST, the membranes were visualized using enhanced chemiluminescence (ECL) prime detection reagents (GE Healthcare, Buckinghamshire, UK). Images were captured using Lumino Image Analyzer LAS-3000 mini (Fujifilm, Tokyo, Japan) and analyzed using ImageJ software (National Institutes of Health, Bethesda, MD). Phosphorylated proteins were normalized to the total amount of each protein.

Vascular permeability assays. Vascular permeability was assessed using CultreCoat 24 well in vitro vascular permeability assay kit (3475-024-K; R\&D systems, Minneapolis, MN). Fresh medium was added to the upper chamber and maintained at $37^{\circ} \mathrm{C}$ in a humidified atmosphere containing $5 \% \mathrm{CO}_{2}$. HUVECs were seeded in the upper chamber $\left(2 \times 10^{6}\right.$ cells/dish $)$ and 500 $\mu \mathrm{l}$ of medium was added to the bottom chamber, then incubated for $72 \mathrm{~h}$. Q-related compounds were then added to the cells in the upper chamber to determine their effects. After $24 \mathrm{~h}$, the medium in the upper chamber was aspirated and the cells were washed with HBSS thrice. Then, the cells in the upper chamber were incubated in medium containing $2 \mathrm{mM} \mathrm{H}_{2} \mathrm{O}_{2}$ for $0.5 \mathrm{~h}$. The medium in the upper chamber was aspirated and the cells were washed with HBSS thrice. The upper chamber was transferred to another 24 well plate and the bottom chamber was filled with $300 \mu \mathrm{l}$ of fresh medium. Fluorescein isothiocyanate (FITC)dextran (3475-096-02) (R\&D systems) was added to the treated cells in the upper chamber and maintained for $5 \mathrm{~min}$. The medium in the bottom chamber was transferred to a 96-well plate and fluorescence (485 $\mathrm{nm}$ excitation with $520 \mathrm{~nm}$ emission) was measured.

Statistical analyses. Data from at least three independent experiments are expressed as mean \pm SD. Statistical analyses were performed using PASW statistics 18.0 (SPSS Inc., Chicago, IL). $P$ value $<0.05$ were considered statistically significant.

\section{Results}

$\mathrm{H}_{2} \mathrm{O}_{2}$ induced Cav-1 phosphorylation. Exposure of HUVECs to $2 \mathrm{mM} \mathrm{H}_{2} \mathrm{O}_{2}$ for 30 min significantly enhanced Cav-1 phosphorylation, although exposure to the same for $15 \mathrm{~min}$ did not show significant enhancement of Cav-1 phosphorylation (Fig. 1A and B). Moreover, $2 \mathrm{mM}$, but not $0.5 \mathrm{mM} \mathrm{H}_{2} \mathrm{O}_{2}$, showed significant phosphorylation of Cav-1 (Fig. 1C and D).

Table 1. Primers used for real-time RT-PCR

\begin{tabular}{lll}
\hline Gene & \multicolumn{1}{c}{ Forward } & \multicolumn{1}{c}{ Reverse } \\
\hline GAPDH & 5'-GCACCGTCAAGGCTGAGAAC-3' & 5'-TGGTGAAGACGCCAGTGGA-3' \\
occludin & 5'-ACAGAGCAAGATCACTATGAGACA-3' & 5'-TGTTGATCTGAAGTGATAGGTGGA-3' \\
JAM & 5'-AAGACACTGGGACATACACTTGT-3' & 5'-CGATGAGCTTGACCTTGACCT-3' $^{\prime}$ \\
ZO-1 & 5'-TGGTGTCCTACCTAATTCAACTCA-3' & 5'-CGCCAGCTACAAATATTCCAACA-3' \\
VE-cadherin & 5'-CAACTGGCCTGTGTTCACGC-3' & 5'-ATCCACTGCTGTCACAGAGATGA-3' \\
\hline
\end{tabular}



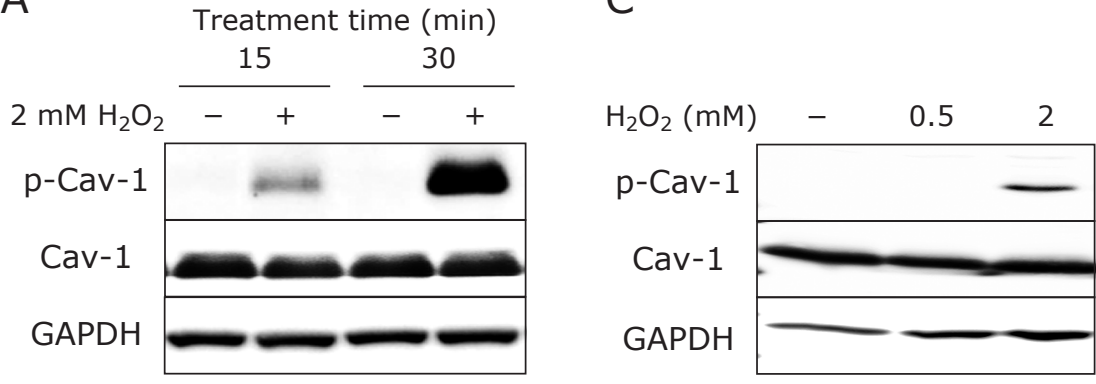

B

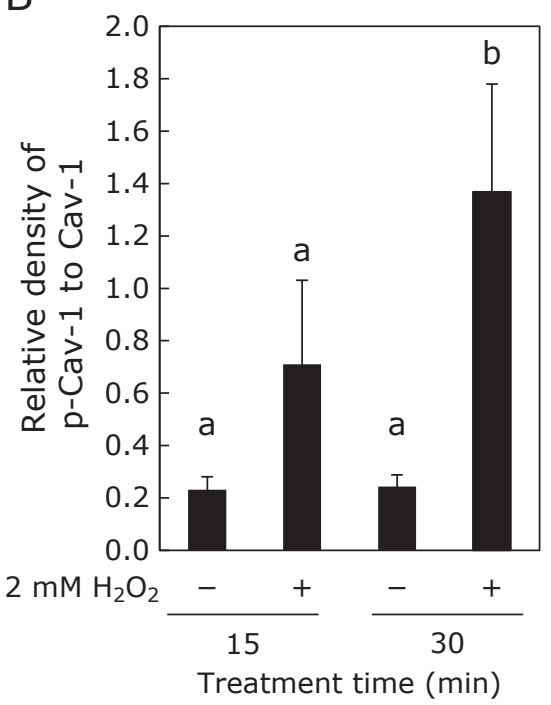

$\mathrm{D}$

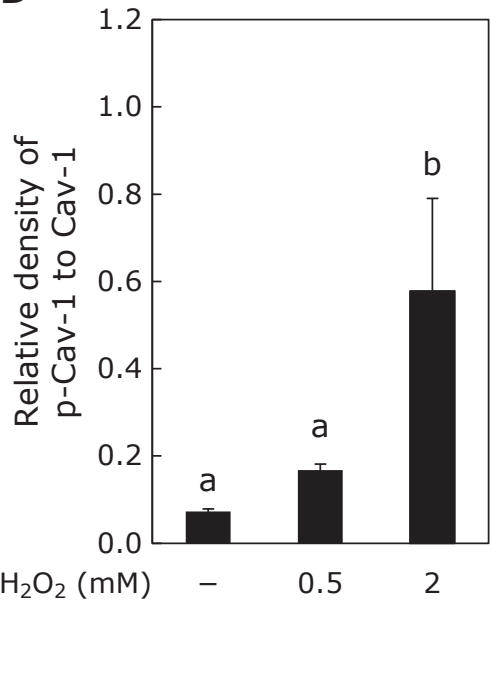

Fig. 1. Enhancement of Cav-1 phosphorylation by $\mathrm{H}_{2} \mathrm{O}_{2}$ in HUVECs. HUVECs were treated with $\mathrm{H}_{2} \mathrm{O}_{2}$ (at 0.5 or $2 \mathrm{mM}$ ) for 15 or 30 min. Phosphorylated Cav-1, total Cav-1, and GAPDH were determined by Western blotting. (A, C) Typical images of Western blotting. (B, D) densities for phosphorylated Cav-1, which were normalized by total Cav-1 obtained from (A), and (C), respectively. Data are means \pm SD ( $n=3$ ). Different letters represent significant differences $(p<0.05)$ determined by ANOVA with the Tukey multiple comparison test.

Suppressive effect of $Q$ and its glucuronide on phosphorylation of Cav-1. Figure 2 shows the effects of $\mathrm{Q}$ and Q3GA on Cav-1 phosphorylation, when HUVECs were treated with these compounds for $0.5 \mathrm{~h}$ (Fig. 2A and B) or $24 \mathrm{~h}$ (Fig. 2C and D) before the addition of $2 \mathrm{mM} \mathrm{H}_{2} \mathrm{O}_{2}$. Q suppressed the phosphorylation of Cav-1 when HUVECs were treated with it for $24 \mathrm{~h}$ before exposure to $\mathrm{H}_{2} \mathrm{O}_{2}$. Q3GA showed no suppression of Cav-1 phosphorylation in the treatment, neither at $0.5 \mathrm{~h}$ nor 24 h. On the contrary, Fyn phosphorylation was unaffected by both Q and Q3GA treatments (Fig. 2C and E). As shown in Fig. 2F, neither Q nor Q3GA had any effect on the phosphorylation of Cav-1 without exposure to $\mathrm{H}_{2} \mathrm{O}_{2}$.

Comparison of suppressive effects of $Q$ analogs on $\mathrm{H}_{2} \mathrm{O}_{2}$ induced Cav-1 phosphorylation. Notably, 8PQ exerted a suppressive effect similar to that of Q, although its suppression was not significantly different. None of the other $\mathrm{Q}$ analogues suppressed the phosphorylation of Cav-1 (Fig. 3).

Effect of $Q$ on $\mathrm{H}_{2} \mathrm{O}_{2}$-induced vascular permeability of HUVECs. To estimate the vascular permeability of HUVECs, they were seeded on a transwell membrane and exposed to $\mathrm{H}_{2} \mathrm{O}_{2}$ for $0.5 \mathrm{~h}$ after pretreatment with $\mathrm{Q}$ for $24 \mathrm{~h}$. Then, the rate of permeation of FITC-dextran in HUVECs was measured by fluorescent assay (Fig. 4). Exposure to $\mathrm{H}_{2} \mathrm{O}_{2}$ increased vascular permeability significantly, while pretreatment with $\mathrm{Q}$ apparently attenuated the $\mathrm{H}_{2} \mathrm{O}_{2}$-induced increase in vascular permeability.

Effect of $Q$ on gene expression related to intercellular junction in HUVECs. Figure 5 shows the effect of $\mathrm{H}_{2} \mathrm{O}_{2}$ exposure for different periods $(0.5,1$, and $3 \mathrm{~h})$ on the expression of genes related to tight junctions and adherens junctions in HUVECs.

The expression of VE-cadherin decreased after 1 and $3 \mathrm{~h}$ of exposure to $\mathrm{H}_{2} \mathrm{O}_{2}$. In contrast, $\mathrm{H}_{2} \mathrm{O}_{2}$ did not affect the expression of occludin, junctional adhesion molecule (JAM), and zonula occludens-1 (ZO-1) after exposure for $0.5,1$, and $3 \mathrm{~h}$. Q showed higher gene expression of VE-cadherin (Fig. 6)

\section{Discussion}

In this study, we investigated the effect of $\mathrm{Q}$ on $\mathrm{H}_{2} \mathrm{O}_{2}$ induced Cav-1 phosphorylation and vascular permeability in endothelial cells. Pre-treatment with Q for $24 \mathrm{~h}$ suppressed $\mathrm{H}_{2} \mathrm{O}_{2}-$ induced Cav-1 phosphorylation and attenuated the enhancement of vascular hyper-permeability (Fig. 2). However, neither the conjugated metabolite of $\mathrm{Q}, \mathrm{Q} 3 \mathrm{GA}$, nor the $\mathrm{Q}$ analogues $\mathrm{L}$, $\mathrm{M}$, and $\mathrm{F}$ suppressed Cav-1 phosphorylation in endothelial cells (Fig. 3). Though there have been few reports about the beneficial effect of flavonoid on phosphorylation of Cav-1, it is demonstrated $\mathrm{Q}$ suppressed phosphorylation of Cav-1 induced by $\mathrm{H}_{2} \mathrm{O}_{2}$ in this study. This is a novel mechanism of $\mathrm{Q}$ on the prevention effect of atherosclerosis.

Fyn, an Src family kinase, plays an essential role in Cav-1 phosphorylation as response to oxidative and hyperosmotic stress. ${ }^{(42)}$ In this study using $\mathrm{H}_{2} \mathrm{O}_{2}$-exposed HUVECs, we confirmed that oxidative stress can induce phosphorylation of both Cav-1 and Fyn (Fig. 1 and 2). $\mathrm{H}_{2} \mathrm{O}_{2}$, a reactive oxygen species, is frequently used at high concentrations (e.g., $2 \mathrm{mM}$ ) to 
A

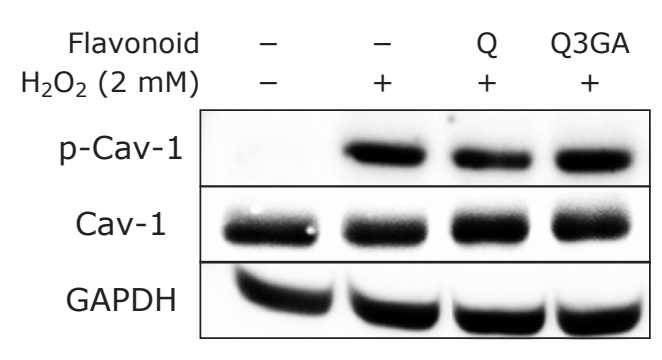

C

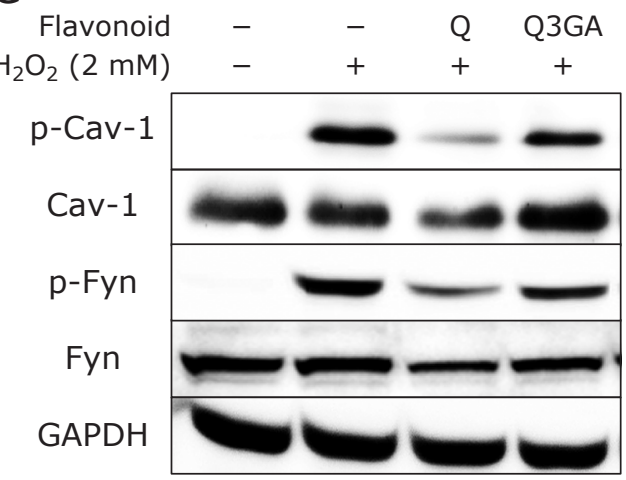

E

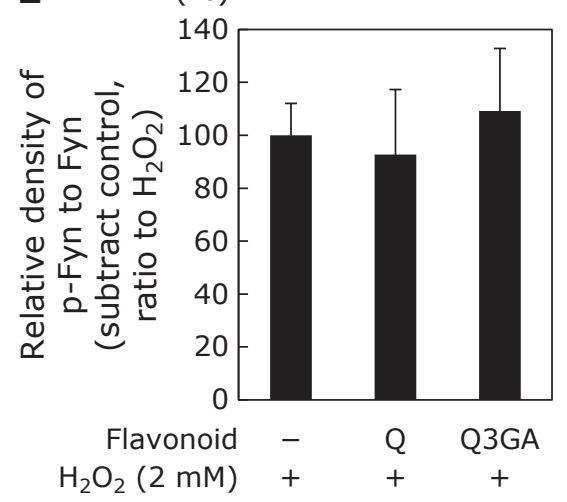

B $(\%)$

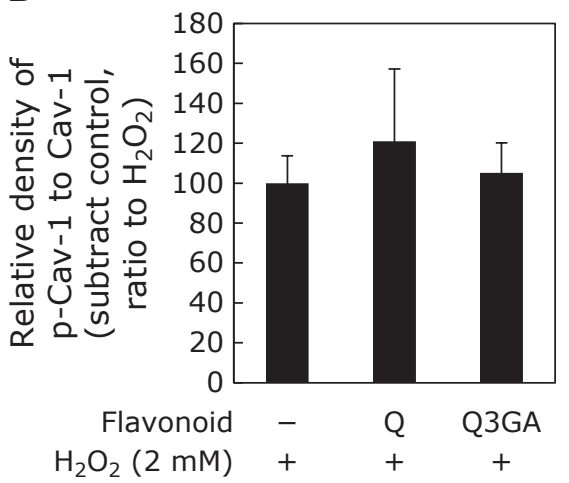

D $\quad(\%)$

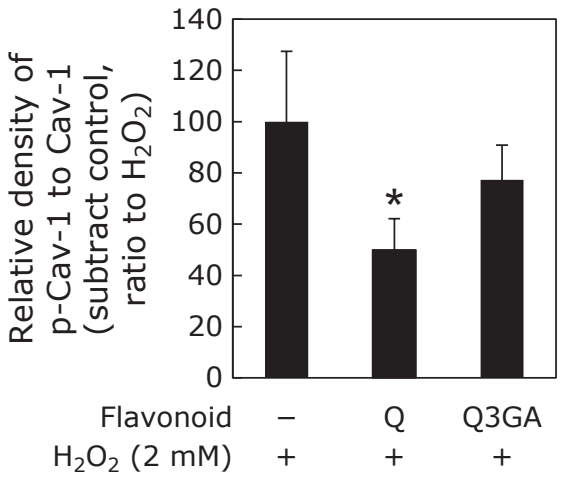

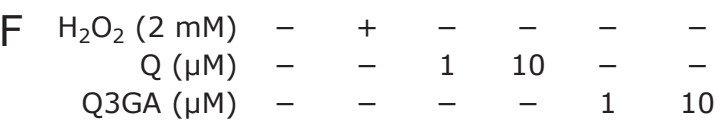
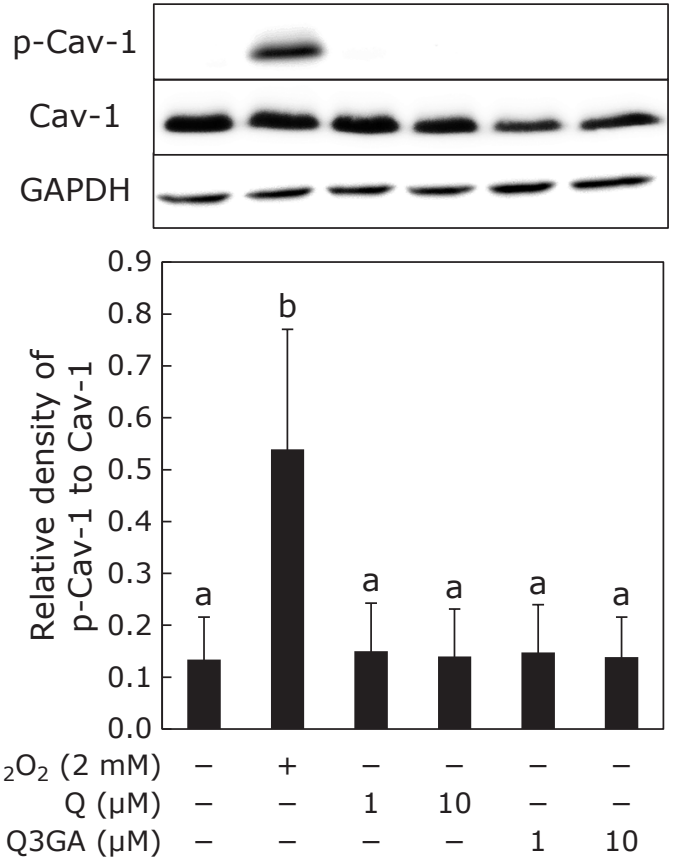

Fig. 2. Effect of $\mathrm{Q}$ and $\mathrm{Q} 3 \mathrm{GA}$ on Cav-1 phosphorylation induced by $\mathrm{H}_{2} \mathrm{O}_{2}$. HUVECs were treated with $\mathrm{Q}$ or Q3GA at $10 \mu \mathrm{M}$ for $0.5 \mathrm{~h}$ (A) or $24 \mathrm{~h}$ (C) prior to the treatment with $2 \mathrm{mM} \mathrm{H}_{2} \mathrm{O}_{2}$ for 30 min. Phosphorylated Cav-1, total Cav-1, phosphorylated Fyn, total Fyn, and GAPDH were determined by Western blotting. (A, C) Typical images of Western blotting. (B, D, E) Densities for phosphorylated protein, which were normalized to each total protein obtained from (A), and (C), respectively. The relative density was calculated as $\%$ of the band density obtained from $\mathrm{H}_{2} \mathrm{O}_{2}$ alone. $\mathrm{HUVECS}$ were treated with Q or Q3GA at 1 or $10 \mu \mathrm{M}$ for $24 \mathrm{~h}(\mathrm{~F}, \mathrm{G})$. Phosphorylated Cav-1, total Cav-1, and GAPDH were determined by Western blotting. (F) Typical images of Western blotting. (G) Densities for phosphorylated Cav-1, which were normalized by total Cav-1 obtained from (F). Data are means $\pm \mathrm{SD}(n=3)$. Asterisks represent significant differences $(p<0.05)$ between $\mathrm{H}_{2} \mathrm{O}_{2}$ alone and each treatment as determined by two-sided Student's $t$ test. 


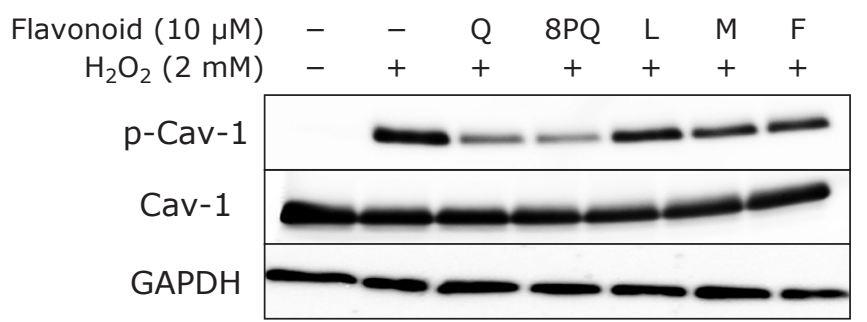

(\%)

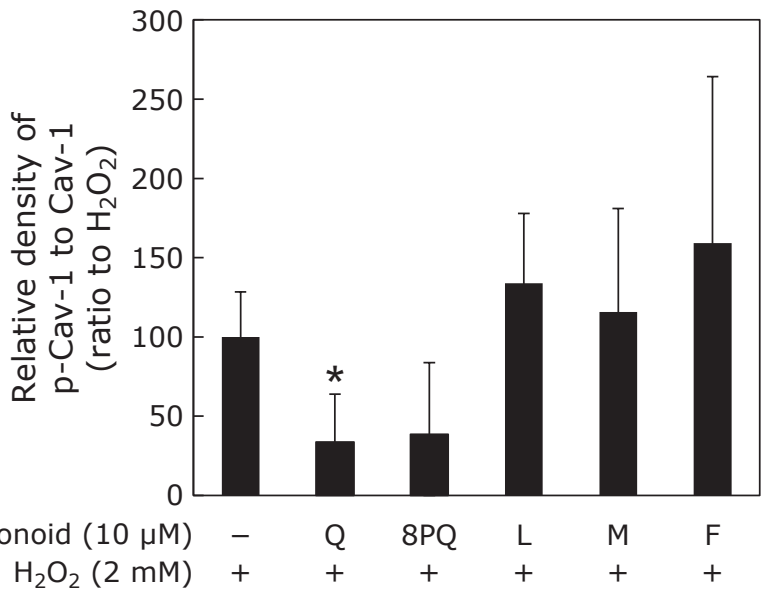

Fig. 3. Effect of $\mathrm{Q}$ analogues on Cav-1 phosphorylation induced by $\mathrm{H}_{2} \mathrm{O}_{2}$. HUVECs were treated with $\mathrm{Q}$ analogues at $10 \mu \mathrm{M}$ for $24 \mathrm{~h}$ prior to treatment with $2 \mathrm{mM} \mathrm{H}_{2} \mathrm{O}_{2}$ for 30 min. Phosphorylated Cav-1, total Cav-1, and GAPDH were determined by Western blotting. (A) Typical images of Western blotting. (B) Densities for phosphorylated protein, which were normalized to each total protein obtained from (A). The relative density was calculated as \% of the band density obtained from $\mathrm{H}_{2} \mathrm{O}_{2}$ alone. Data are means $\pm \mathrm{SD}(n=3)$. Asterisk represents significant differences $(p<0.05)$ between $\mathrm{H}_{2} \mathrm{O}_{2}$ alone and each treatment, as determined by two-sided Student's $t$ test.

induce oxidative stress in cultured cells, including HUVECs. ${ }^{(43)}$ According to the study of Shin et al. ${ }^{(44)}$ the addition of $1 \mu \mathrm{M}$ sodium orthovanadate to the cells inhibited cellular phosphatase activity. Therefore, the cellular system used in this study can reflect the oxidative stress in vascular endothelial cells in vivo.

We demonstrated that pretreatment of HUVECs with Q for $24 \mathrm{~h}$ exerted inhibitory effect on Cav-1 phosphorylation in these cells (Fig. 2). The inhibitory effect of Q on Cav-1 phosphorylation can be explained by both its direct antioxidant activity and indirect activity responsible for the cellular signal transduction pathway. In contrast, Q did not show any effect on Fyn phosphorylation (Fig. 2). Fyn is phosphorylated in the cytoplasm, ${ }^{(45)}$ while Cav-1 phosphorylation occurs at the caveolae in the plasma membrane of vascular endothelial cells. ${ }^{(46)}$ It is therefore likely that $\mathrm{Q}$ is capable of acting at caveolae in the plasma membrane, but not in the cytoplasm. Although few studies have demonstrated the cellular localization of Q in HUVECs, Q hardly existed in cultured cells after $24 \mathrm{~h}$ of treatment. ${ }^{(47,48)}$ Furthermore, direct antioxidant activity is unlikely to be responsible for cardiovascular health in humans. ${ }^{(49)}$ Therefore, we suppose that $Q$ may affect intracellular signaling pathways to exert its inhibitory effect. Piechota-Polanczyk et al. ${ }^{(50)}$ showed that heme oxygenase-1 (HO-1), an intracellular antioxidant enzyme, is involved in the inhibition of Cav-1 phosphorylation in a mouse model. Q is known to increase HO-1 gene expression via phosphorylation of ERK, which inhibits $\mathrm{H}_{2} \mathrm{O}_{2}$-induced apoptosis. ${ }^{(51)}$ Therefore, $\mathrm{Q}$ may act as an inhibitor of Cav-1 phosphorylation through

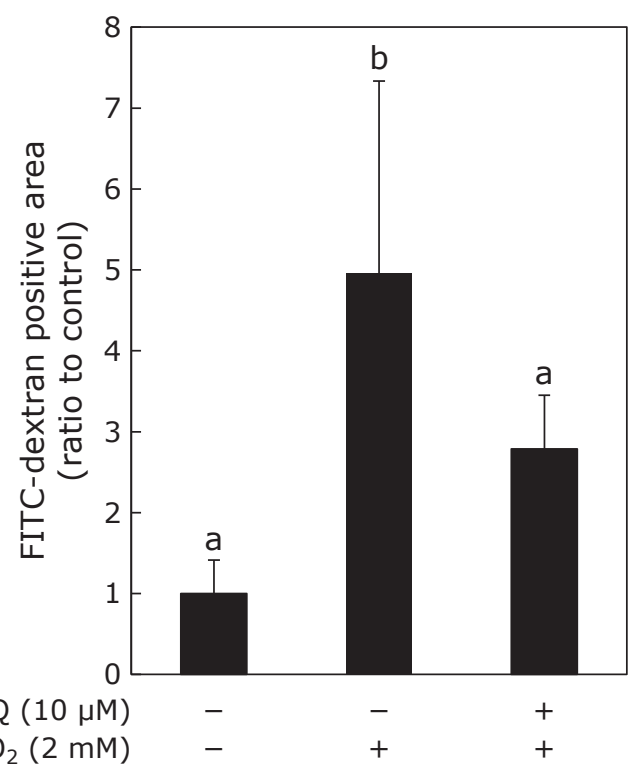

Fig. 4. Suppressive effect of $Q$ on vascular permeability. HUVECs seeded on the transwell were treated with $10 \mu \mathrm{M} \mathrm{Q}$ for $24 \mathrm{~h}$ prior to treatment with $2 \mathrm{mM} \mathrm{H}_{2} \mathrm{O}_{2}$ for $30 \mathrm{~min}$. Vascular permeability was estimated by the measurement of fluorescent FITC-Dextran that passes through HUVEC cell. The index was calculated based on the ratio between control and independent experiments. Data are means \pm SD $(n=3)$. Different letters represent significant differences $(p<0.05)$ determined by ANOVA with the Tukey multiple comparison test.

the induction of HO-1 expression. ${ }^{(50)}$ Nuclear accumulation of $\mathrm{Nrf2}$, a transcription factor for the gene expression of HO-1, and downregulation of the Nrf2 repressor, Keap1, reportedly participate in Q-dependent HO-1 induction. ${ }^{(52)}$ Therefore, it can be hypothesized that HO-1 induction is involved in the inhibition of Cav-1 phosphorylation when HUVECs are pretreated with $\mathrm{Q}$ for $24 \mathrm{~h}$. In HUVECs, AMPK stabilizes Prdx 1 and c-Abl complexes, thus acting as a negative regulator of Cav-1 phosphorylation. ${ }^{(43)}$

Cav-1 knockdown markedly reduced the localization of VEcadherin and $\beta$-catenin at the endothelial cell binding sites. ${ }^{(53)}$ Exposure to $\mathrm{H}_{2} \mathrm{O}_{2}$ causes dissociation of $\beta$-catenin from Cav-1 and VE-cadherin. ${ }^{(23)}$ Our results revealed that $\mathrm{H}_{2} \mathrm{O}_{2}$ exacerbated vascular permeability, concomitant with both a decrease in VEcadherin mRNA expression and Cav-1 phosphorylation. These phenomena indicate that vascular permeability is increased by the deficiency of cell-cell adhesion proteins under oxidative stress. Thus, Q appears to contribute to the maintenance of vascular function by suppressing the attenuation of VE-cadherin gene expression through inhibition of oxidative stress dependentCav-1 phosphorylation.

The results of the structure-activity relationship study indicated that the $\mathrm{Q}$ analogues tested in this study did not exert inhibitory effects on Cav-1 phosphorylation (Fig. 3). The inherent structure of Q, a flavonol structure with an odihydroxy group at the B-ring, may be necessary for exerting this activity. Interestingly, the present study did not show any inhibitory effects of Q3GA (Fig. 2). Q is present as conjugated glucuronide and/or sulfate metabolites in blood. ${ }^{(29)}$ It is therefore implied that deconjugation and conversion into $\mathrm{Q}$ aglycone by $\beta$-glucuronidase at the site of vascular inflammation is required for $\mathrm{Q}$ glucuronide conjugates to exert their vascular function through modulation of Cav-1 phosphorylation in vivo.

Q mainly exists as glycosylated form such as its glucoside or rutinoside in natural sources. ${ }^{(54)}$ Recent studies using rodents demonstrated that these glycosides exerted beneficial effect on 
A

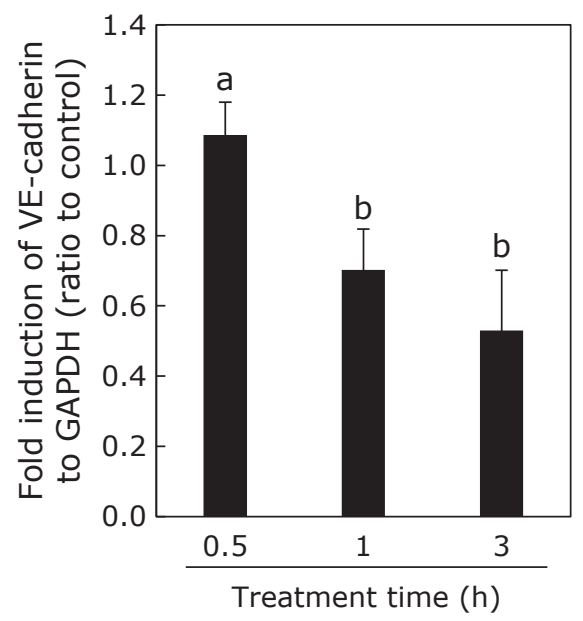

C

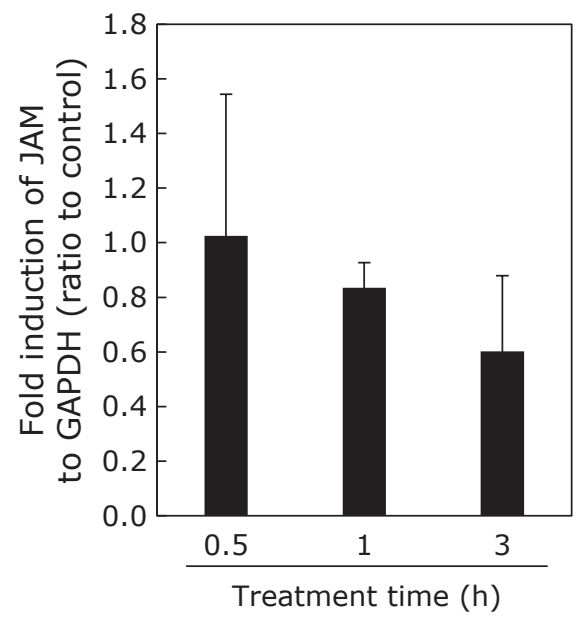

B

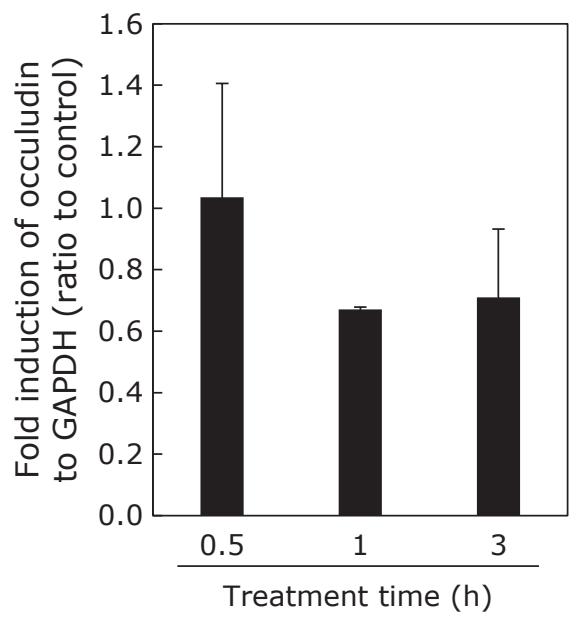

D

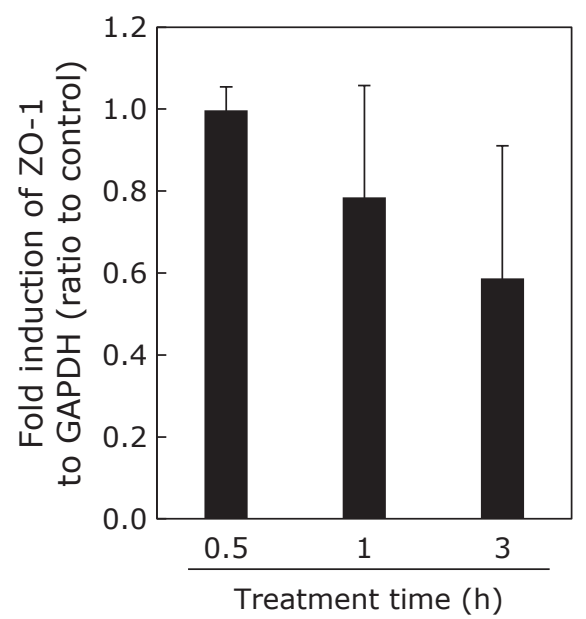

Fig. 5. Effect of $\mathrm{H}_{2} \mathrm{O}_{2}$ on vascular permeability related gene expression. HUVECs were treated with 2 mM $\mathrm{H}_{2} \mathrm{O}_{2}$ for 0.5 , 1 , or 3 h. Gene expression was normalized to that of GAPDH expression. Data were calculated as the ratio between control (without $\mathrm{H}_{2} \mathrm{O}_{2}$ treatment) and treated cells. Data are means \pm SD $(n=3)$. Different letters represent significant differences $(p<0.05)$ determined by ANOVA with the Tukey multiple comparison test.

hyperglycemia and blood stasis syndrome, ${ }^{(55,56)}$ when they were orally administered. These glycosides are hydrolyzed in the intestinal tract and resulting aglycone is transferred into the blood stream. ${ }^{(57)}$ Therefore, Q glycosides from dietary origin seem to possess a potential to exert preventive effect on exacerbation of vascular permeability caused by oxidative stress.

In conclusion, we confirmed that $\mathrm{H}_{2} \mathrm{O}_{2}$ causes phosphorylation of Cav-1, reduces the mRNA expression of the plasma membrane-bound protein VE-cadherin, and increases vascular permeability in HUVECs. Q aglycone suppresses both $\mathrm{H}_{2} \mathrm{O}_{2}$ dependent Cav-1 phosphorylation and increases vascular permeability. Q may protect the vascular endothelium by suppressing the phosphorylation of Cav-1 in the endothelial cells under oxidative stress. Q increased VE-cadherin expression under $\mathrm{H}_{2} \mathrm{O}_{2}$ exposure (Fig. 6C). Further study is needed for clarify whether Q protect VE-cadherin down-regulation by $\mathrm{H}_{2} \mathrm{O}_{2}$ through suppression of Cav-1 phosphorylation or not. Q glucuronide metabolite did not show such an inhibitory effect, indicating that the release of aglycone from a plasma-conjugated metabolite is necessary for dietary $\mathrm{Q}$ to exert vascular function.

\section{Author Contributions}

Study concept and design (JT, TS, and RM); acquisition of data and statistical analysis (AKK and RM); analysis and interpretation of data, drafting of the manuscript, obtained funding (AKK, JT, and RM); critical version of the manuscript for important intellectual content (JT and RM); obtained funding (AKK, JT, and RM); and study supervision (JT and TS).

\section{Acknowledgments}

This work was supported by the Tojuro Iijima Foundation for Food Science and Technology, and was partly supported by JSPS KAKENHI Grant Numbers 25292075 and 17K13213.

\section{Abbreviations}

BCA

Cav-1

ECL $\mathrm{F}$

FITC bicinchoninic acid caveolin-1 enhanced chemiluminescence flavone fluorescein isothiocyanate 
A

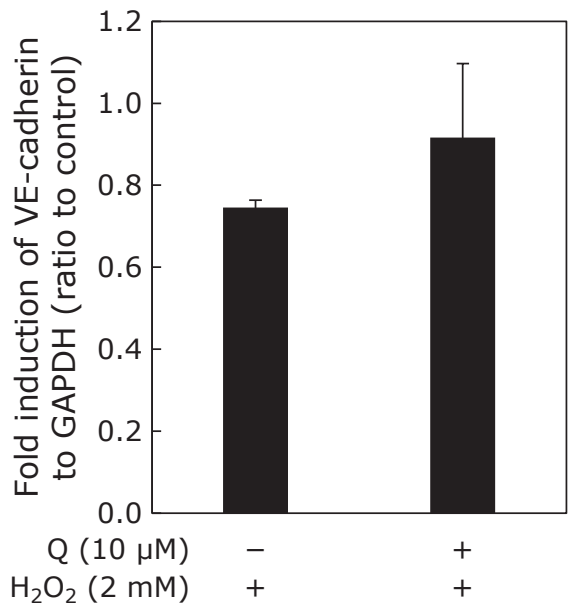

C

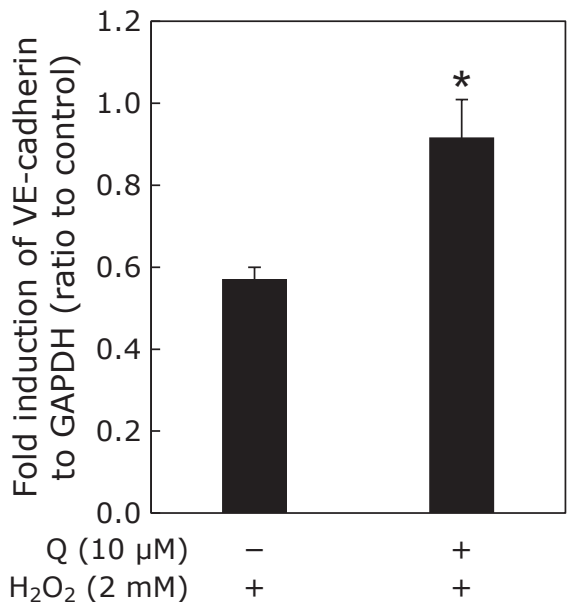

B

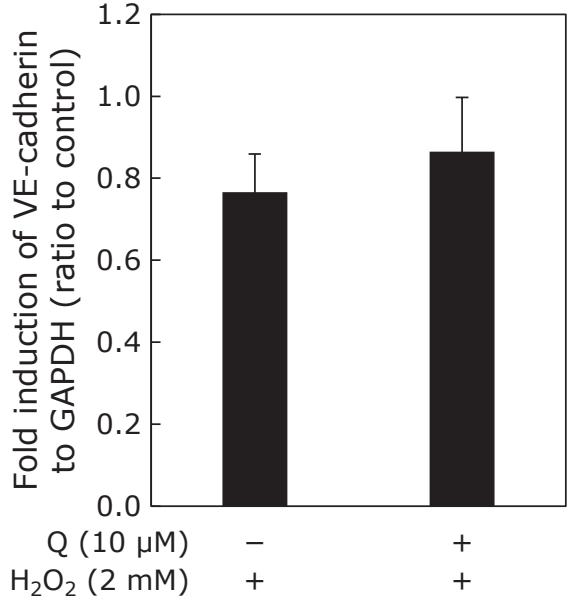

Fig. 6. Effect of $\mathrm{Q}$ on vascular permeability-related gene expression in the presence of $\mathrm{H}_{2} \mathrm{O}_{2}$. $\mathrm{HUVECs}$ were treated with $10 \mu \mathrm{M} \mathrm{Q}$ for $24 \mathrm{~h}$ prior to treatment with $2 \mathrm{mM} \mathrm{H}_{2} \mathrm{O}_{2}$ for 0.5 , 1 , or $3 \mathrm{~h}$. Gene expression was normalized to that of GAPDH expression. Data were calculated as the ratio to control (without $\mathrm{H}_{2} \mathrm{O}_{2}$ treatment). Data are means $\pm \mathrm{SD}(n=3)$. Asterisk represents significant differences $(p<0.05)$ between control $\left(\mathrm{H}_{2} \mathrm{O}_{2}\right.$ alone) and $\mathrm{Q}$ by two-sided Student's $t$ test.

$\begin{array}{ll}\text { GAPDH } & \text { glyceraldehyde-3-phosphate dehydrogenase } \\ \mathrm{H}_{2} \mathrm{O}_{2} & \text { hydrogen peroxide } \\ \mathrm{HUVEC} & \text { human umbilical vein endothelial cells } \\ \text { JAM } & \text { junctional adhesion molecule } \\ \text { L } & \text { luteolin } \\ \text { M } & \text { myricetin } \\ \text { OX-LDL } & \text { oxidized low-density proteins } \\ \text { 8PQ } & \text { 8-prenyl quercetin } \\ \text { Q } & \text { quercetin }\end{array}$

\section{References}

1 Roth GA, Johnson C, Abajobir A, et al. Global, regional, and national burden of cardiovascular diseases for 10 causes, 1990 to 2015. J Am Coll Cardiol 2017; 70: 1-25.

2 Beaglehole R, Yach D. Globalisation and the prevention and control of noncommunicable disease: the neglected chronic diseases of adults. Lancet 2003; 362: 903-908.

3 Mozaffarian D. Global scourge of cardiovascular disease: time for health care systems reform and precision population health. J Am Coll Cardiol 2017; 70: $26-28$.

$\begin{array}{ll}\text { Q3GA } & \text { quercetin 3-O-glucuronide } \\ \text { RT-PCR } & \text { reverse-transcription polymerase chain reaction } \\ \text { SDS } & \text { sodium dodecyl sulfate polyacrylamide } \\ \text { VE-cadherin } & \text { vascular endothelial cadherin } \\ \text { ZO-1 } & \text { zonula occludens-1 }\end{array}$

\section{Conflict of Interest}

No potential conflicts of interest were disclosed.
4 Madamanchi NR, Vendrov A, Runge MS. Oxidative stress and vascular disease. Arterioscler Thromb Vasc Biol 2005; 25: 29-38.

5 Stone NJ, Robinson JG, Lichtenstein AH, et al. 2013 ACC/AHA guideline on the treatment of blood cholesterol to reduce atherosclerotic cardiovascular risk in adults: a report of the American College of Cardiology/American Heart Association Task Force on Practice Guidelines. J Am Coll Cardiol 2014; 63 (25 Pt B): 2889-2934.

6 Torres N, Guevara-Cruz M, Velázquez-Villegas LA, Tovar AR. Nutrition and Atherosclerosis. Arch Med Res 2015; 46: 408-426. 
7 Ruiz-León AM, Lapuente M, Estruch R, Casas R. Clinical advances in immunonutrition and atherosclerosis: a review. Front Immunol 2019; 10: 837.

8 Lum H, Malik AB. Regulation of vascular endothelial barrier function. Am J Physiol 1994; 267 (3 Pt 1): L223-L241.

9 Bazzoni G, Dejana E. Endothelial cell-to-cell junctions: molecular organization and role in vascular homeostasis. Physiol Rev 2004; 84: 869901.

10 Mehta D, Malik AB. Signaling mechanisms regulating endothelial permeability. Physiol Rev 2006; 86: 279-367.

11 Murata T, Lin MI, Aritake K, et al. Role of prostaglandin D2 receptor DP as a suppressor of tumor hyperpermeability and angiogenesis in vivo. Proc Natl Acad Sci U S A 2008; 105: 20009-20014.

12 Lisanti MP, Scherer PE, Tang Z, Sargiacomo M. Caveolae, caveolin and caveolin-rich membrane domains: a signalling hypothesis. Trends Cell Biol 1994; 4: 231-235.

13 Razani B, Lisanti M. The role of caveolae and the caveolins in mammalian physiology Rice Undergraduate Science Review 2002; 1: 44-45.

14 Layne J, Majkova Z, Smart EJ, Toborek M, Hennig B. Caveolae: a regulatory platform for nutritional modulation of inflammatory diseases. $J$ Nutr Biochem 2011; 22: 807-811.

15 Schnitzer JE, Oh P, McIntosh DP. Role of GTP hydrolysis in fission of caveolae directly from plasma membranes. Science 1996; 274: 239-242.

16 Liu P, Rudick M, Anderson RG. Multiple functions of caveolin-1. J Biol Chem 2002; 277: 41295-41298.

17 Pavlides S, Gutierrez-Pajares JL, Iturrieta J, Lisanti MP, Frank PG. Endothelial caveolin-1 plays a major role in the development of atherosclerosis. Cell Tissue Res 2014; 356: 147-157.

18 Fernández-Hernando C, Yu J, Dávalos A, Prendergast J, Sessa WC Endothelial-specific overexpression of caveolin-1 accelerates atherosclerosis in apolipoprotein E-deficient mice. Am J Pathol 2010; 177: 998-1003.

$19 \mathrm{Hu}$ G, Vogel SM, Schwartz DE, Malik AB, Minshall RD. Intercellular adhesion molecule-1-dependent neutrophil adhesion to endothelial cells induces caveolae-mediated pulmonary vascular hyperpermeability. Circ Res 2008; 102: e120-e131.

$20 \mathrm{Hu} \mathrm{G}$, Minshall RD. Regulation of transendothelial permeability by Src Kinase. Microvasc Res 2009; 77: 21-25.

21 Zhang Y, Zhang L, Li Y, Sun S, Tan H. Different contributions of clathrin- and caveolae-mediated endocytosis of vascular endothelial cadherin to lipopolysaccharide-induced vascular hyperpermeability. PLoS One 2014; 9: e106328.

22 Lin F, Pei L, Zhang Q, et al. Ox-LDL induces endothelial cell apoptosis and macrophage migration by regulating caveolin-1 phosphorylation. $J$ Cell Physiol 2018; 233: 6683-6692.

23 Sun Y, Hu G, Zhang X, Minshall RD. Phosphorylation of caveolin-1 regulates oxidant-induced pulmonary vascular permeability via paracellular and transcellular pathways. Circ Res 2009; 105: 676-685.

24 Miean KH, Mohamed S. Flavonoid (myricetin, quercetin, kaempferol, luteolin, and apigenin) content of edible tropical plants. J Agric Food Chem 2001; 49: 3106-3112.

25 Aherne SA, O'Brien NM. Dietary flavonols: chemistry, food content, and metabolism. Nutrition 2002; 18: 75-81.

26 Landberg R, Naidoo N, van Dam RM. Diet and endothelial function: from individual components to dietary patterns. Curr Opin Lipidol 2012; 23: 147 155 .

27 Hertog MG, Hollman PC, Katan MB, Kromhout D. Intake of potentially anticarcinogenic flavonoids and their determinants in adults in The Netherlands. Nutr Cancer 1993; 20: 21-29.

28 Hertog MG, Kromhout D, Aravanis C, et al. Flavonoid intake and long-term risk of coronary heart disease and cancer in the seven countries study. Arch Intern Med 1995; 155: 381-386.

29 Terao J. Factors modulating bioavailability of quercetin-related flavonoids and the consequences of their vascular function. Biochem Pharmacol 2017; 139: $15-23$.

30 Salvamani S, Gunasekaran B, Shaharuddin NA, Ahmad SA, Shukor MY. Antiartherosclerotic effects of plant flavonoids. Biomed Res Int 2014; 2014: 480258.

31 Deng Q, Li XX, Fang Y, Chen X, Xue J. Therapeutic potential of quercetin as an antiatherosclerotic agent in atherosclerotic cardiovascular disease: a review. Evid Based Complement Alternat Med 2020; 2020: 5926381.

32 Starke H, Herrmann K. Flavonols and flavones of vegetables. VI. On the changes of the flavonols of onions (authors transl). Z Lebensm Unters Forsch 1976; 161: 137-142. (in Deutsch)

33 Day AJ, Cañada FJ, Díaz JC, et al. Dietary flavonoid and isoflavone glycosides are hydrolysed by the lactase site of lactase phlorizin hydrolase. FEBS Lett 2000; 468: 166-170.

34 Kawai Y, Nishikawa T, Shiba Y, et al. Macrophage as a target of quercetin glucuronides in human atherosclerotic arteries: implication in the anti-atherosclerotic mechanism of dietary flavonoids. J Biol Chem 2008; 283 : 9424-9434.

35 Ishisaka A, Kawabata K, Miki S, et al. Mitochondrial dysfunction leads to deconjugation of quercetin glucuronides in inflammatory macrophages. PLoS One 2013; 8: e80843.

36 Menendez C, Dueñas M, Galindo $\mathrm{P}$, et al. Vascular deconjugation of quercetin glucuronide: the flavonoid paradox revealed? Mol Nutr Food Res 2011; 55: 1780-1790.

37 Galindo P, Rodriguez-Gómez I, González-Manzano S, et al. Glucuronidated quercetin lowers blood pressure in spontaneously hypertensive rats via deconjugation. PLoS One 2012; 7: e32673.

38 Perez A, Gonzalez-Manzano S, Jimenez R, et al. The flavonoid quercetin induces acute vasodilator effects in healthy volunteers: correlation with betaglucuronidase activity. Pharmacol Res 2014; 89: 11-18.

39 Kamada C, Mukai R, Kondo A, Sato S, Terao J. Effect of quercetin and its metabolite on caveolin-1 expression induced by oxidized LDL and lysophosphatidylcholine in endothelial cells. J Clin Biochem Nutr 2016; 58 193-201.

40 Kawamura T, Hayashi M, Mukai R, Terao J, Nemoto H. An efficient method for C8-prenylation of flavonols and flavanones. Synthesis 2012; 44: 13081314.

41 Mukai R, Nakao R, Yamamoto H, Nikawa T, Takeda E, Terao J. Quercetin prevents unloading-derived disused muscle atrophy by attenuating the induction of ubiquitin ligases in tail-suspension mice. J Nat Prod 2010; 73: $1708-1710$

42 Sanguinetti AR, Cao H, Corley Mastick C. Fyn is required for oxidative- and hyperosmotic-stress-induced tyrosine phosphorylation of caveolin-1. Biochem $J$ 2003; 376 (Pt 1): 159-168.

43 Takeuchi K, Morizane Y, Kamami-Levy C, et al. AMP-dependent kinase inhibits oxidative stress-induced caveolin-1 phosphorylation and endocytosis by suppressing the dissociation between c-Abl and $\operatorname{Prdx} 1$ proteins in endothelial cells. J Biol Chem 2013; 288: 20581-20591.

44 Shin J, Jo H, Park H. Caveolin-1 is transiently dephosphorylated by shear stress-activated protein tyrosine phosphatase mu. Biochem Biophys Res Commun 2006; 339: 737-741.

45 Resh MD. Fyn, a Src family tyrosine kinase. Int J Biochem Cell Biol 1998; 30: $1159-1162$.

46 Lee H, Volonte D, Galbiati F, et al. Constitutive and growth factor-regulated phosphorylation of caveolin-1 occurs at the same site (Tyr-14) in vivo: identification of a c-Src/Cav-1/Grb7 signaling cassette. Mol Endocrinol 2000; 14: $1750-1775$

47 Mukai R, Fujikura Y, Murota K, et al. Prenylation enhances quercetin uptake and reduces efflux in Caco-2 cells and enhances tissue accumulation in mice fed long-term. J Nutr 2013; 143: 1558-1564.

48 Mukai R, Kawabata K, Otsuka S, et al. Effect of quercetin and its glucuronide metabolite upon 6-hydroxydopamine-induced oxidative damage in Neuro-2a cells. Free Radic Res 2012; 46: 1019-1028.

49 Hollman PCH, Cassidy A, Comte B, et al. The biological relevance of direct antioxidant effects of polyphenols for cardiovascular health in humans is not established. J Nutr 2011; 141: 989S-1009S.

50 Piechota-Polanczyk A, Zielińska M, Piekielny D, Fichna J. The influence of lipoic acid on caveolin-1-regulated antioxidative enzymes in the mouse model of acute ulcerative colitis. Biomed Pharmacother 2016; 84: 470-475.

51 Chow JM, Shen SC, Huan SK, Lin HY, Chen YC. Quercetin, but not rutin and quercitrin, prevention of $\mathrm{H}_{2} \mathrm{O}_{2}$-induced apoptosis via anti-oxidant activity and heme oxygenase 1 gene expression in macrophages. Biochem Pharmacol 2005; 69: 1839-1851.

52 Luo M, Tian R, Yang Z, Peng YY, Lu N. Quercetin suppressed NADPH oxidase-derived oxidative stress via heme oxygenase-1 induction in macrophages. Arch Biochem Biophys 2019; 671: 69-76.

53 Song L, Ge S, Pachter JS. Caveolin-1 regulates expression of junctionassociated proteins in brain microvascular endothelial cells. Blood 2007; 109: $1515-1523$. 
54 Tsushida T, Suzuki M. Isolation of flavonoid-glycosides in onion and identification by chemical synthesis of the glycosides flavonoid in fruits and vegetables Part I. Nippon Shokuhin Kagaku Kogaku Kaishi 1995; 42: 100108. (in Japanese)

$55 \mathrm{Oku} \mathrm{H}$, Maeda M, Kitagawa F, Ishiguro K. Effect of polyphenols from syringa vulgaris on blood stasis syndrome. J Clin Biochem Nutr 2020; 67: 84-88.

56 Jiang H, Horiuchi Y, Hironao KY, Kitakaze T, Yamashita Y, Ashida H. Prevention effect of quercetin and its glycosides on obesity and hyperglycemia through activating AMPK $\alpha$ in high-fat diet-fed ICR mice. $J$
Clin Biochem Nutr 2020; 67: 74-83.

57 Murota K, Terao J. Antioxidative flavonoid quercetin: implication of its intestinal absorption and metabolism. Arch Biochem Biophys 2003; 417: 12 17.

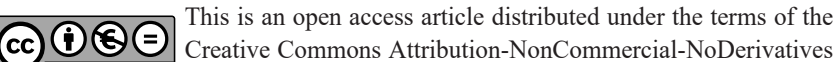
BY NC ND License (http://creativecommons.org/licenses/by-nc-nd/4.0/). 